\title{
CONTROLE QUIMÍCO DA PLANTA AQUÁTICA ANCORRADA ERVA DO PÂNTANO (Enydra sessilis)
}

\section{CHEMICAL CONTROL OF ANCHORED AQUATIC PLANT SWAMP HERB (Enydra sessilis)}

\author{
Wilson Roberto Cerveira Junior ${ }^{1,2}$, Pâmela Castro Pereira ${ }^{1,2}$, Claudinei da Cruz ${ }^{2}$ \\ ${ }^{1}$ Laboratório de Ecotoxicologia e Eficácia dos Agrotóxicos, LEEA, do Centro Universitário da Fundação \\ Educacional de Barretos, SP, Brasil. \\ ${ }^{2}$ Faculdade de Ciências Agrárias e Veterinárias, Unesp, Jaboticabal, SP, Brasil.
}

\section{RESUMO}

As plantas aquáticas de transição entre o ambiente aquático e terrestre desempenham papel fundamental no equilíbrio entres os ambientes. O objetivo deste estudo foi avaliar a eficácia de controle dos herbicidas imazamox e diquat no controle da erva de pântano (Enydra sessilis). As doses utilizadas no presente estudo foram 200,0, 400,0, 600,0 e 800 g i.a. ha ${ }^{-1}$ para o herbicida imazamox e 100,0, 200,0, 300,0 e 400 g i.a. ha ${ }^{-1}$ para o diquat. $\mathrm{O}$ experimento foi conduzido em casa de vegetação e as avaliações foram realizadas aos $3,7,15,21,30,45$ e 60 dias após a aplicação (DAA). Os resultados evidenciaram que o herbicida imazamox apresentou um controle bom apenas na dose de $800,0 \mathrm{~g}$ i.a. ha ${ }^{-1}$, enquanto o diquat apresentou $100 \%$ de controle em todas as doses testadas. Conclui-se que o herbicida diquat apresentou um melhor controle para a erva de pântano enquanto o imazamox varia o controle de acordo com a dose utilizada.

Palavras-chave: Várzea, Macrófita, Herbicida

\begin{abstract}
Aquatic plants in transition between the aquatic and terrestrial environment play a fundamental role in the balance between environments. The objective of this study was to evaluate the control effectiveness of the herbicides imazamox and diquat in the control of swamp grass (Enydra sessilis). The doses used in the

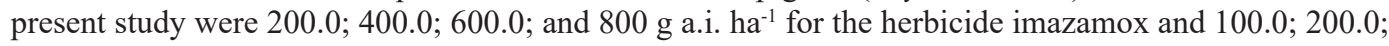
300.0 ; and $400 \mathrm{~g}$ a.i. ha ${ }^{-1}$ for the diquat. The experiment was conducted in a greenhouse and the evaluations were carried out at $3,7,15,21,30,45$, and 60 days after application (DAA). The results showed that the herbicide imazamox had good control only at the dose of $800.0 \mathrm{~g}$ a.i. ha ${ }^{-1}$, while diquat had $100 \%$ control at the lowest dose tested. It was concluded that the herbicide diquat showed a better control for swamp grass while imazamox varies the control according to the dose used.
\end{abstract}

Keywords: Floodplain, Macrophyte, Herbicide

Autor para correspondência: Wilson Roberto Cerveira Junior, endereço: Avenida Professor Roberto Frade Monte, n. 389, Barretos, SP. E-mail: wilsonrcjunior@gmail.com

Recebido para publicação: $01 /$ jun./2020

Aceito para publicação: 17/jun./2020

https://doi.org/10.4322/1980-0029.182020 


\section{Introdução}

No Brasil, estima-se que cerca de $6 \%$ do território nacional seja coberto por áreas alagáveis, o que, somado às características favoráveis das regiões tropicais, favorece a ocorrência de várias espécies de plantas aquáticas na transição do ambiente terrestre e aquático, tanto em ambientes aquáticos naturais como artificiais, sendo os gêneros Eichhornia sp., Eleocharis sp., Enhydra sp., Ludwigia sp., Oxycaryum cubense e Typha dominguensis (Bianchini Junior et al., 2010), Echinochloa polystachya, Panicum repens, Polygonum acuminatum e Polygonum ferrugineum (Amorim et al., 2015), além de plantas invasoras como a Brachiaria subquadripara, Hedychium coronarium e Myriophyllum aquaticum (Cerveira Junior et al., 2019).

As plantas aquáticas de transição entre o ambiente aquático e terrestre desempenham papel fundamental no equilíbrio entres os ambientes, com regulação do seu equilíbrio de trocas de nutrientes e matéria orgânica. As comunidades de plantas destas regiões são estruturadas para atuarem como filtro ambiental (Keddy, 1992). Os filtros ambientais selecionam aqueles organismos que apresentam conjuntos de características ou estratégias que melhor se ajustam às possíveis populações (Baastrup-Spohr et al., 2015).

O maior problema das ocupações por colonizações monoespecíficas de plantas aquáticas dos ambientes de transição é a perda de habitats e biodiversidade, pois estes fornecem uma variedade de serviços ecossistêmicos, como controlar inundações, regular o clima regional e fornecer água potável e água agrícola (Lui et al., 2006). Para redução dos prejuízos causados pelas invasões das plantas aquáticas é necessário o desenvolvimento de um plano de manejo. O controle químico apresenta excelente eficácia e boa relação custo/benefício (Gettys et al., 2014) e no Brasil ocorreu a aprovação e regulamentação, por meio da Resolução n ${ }^{\circ} 467$, que dispõe sobre o uso de produtos ou processos para recuperação de ambientes hídricos (Conselho Nacional do Meio Ambiente, 2015).

Alguns herbicidas se destacam no controle de plantas aquáticas flutuantes ou emergências enraizadas, tais como: o ethoxysulfuron, oxadiargyl e idiosulfuron para Enhydra fluctuans e outras plantas invasoras do cultivo de arroz (Hasanuzzaman et al., 2009); o diquat para Eichhornia crassipes, Schoenoplectus californicus e Pistia stratiotes
(Mudge \& Netherland, 2014); o imazapyr para E. crassipes, P. stratiotes e Salvinia molesta (Cruz et al., 2015a); o glyphosate para $S$. molesta, S. herzogui, P. stratiotes, Urochloa subquadripara e E. crassipes (Cruz et al., 2015b); o triclopyr para Miriophyllum aquaticum (Hofstra et al., 2006); o arylpicolinate para Alternanthera philoxeroides (Richardson et al., 2016); o glyphosate para Salvinia herzogii, S. molesta e S. oblongifolia em aplicação sequencial (Cruz et al., 2017); o glyphosate isolado e com os adjuvantes Aterbane ${ }^{\circledR}$ BR, Veget'oil ${ }^{\circledR} \mathrm{e}$ Dash $^{\circledR} \mathrm{HC}$ e pontas de pulverização (AI 110.02, TTI 110.015 e TT 110.015$)$ para $P$. stratiotes (Silva et al., 2018); e o imazamox com bico de pulverização de energia centrífuga para $E$. crassipes (Garlich et al., 2019).

Dentre os herbicidas com potencial para o controle de plantas aquáticas, algumas moléculas têm sido testadas, como o diquat e imazamox. $\mathrm{O}$ diquat (1-1'-etileno-2-2'-dibrometo de bipiridílio) é um herbicida de contato, não seletivo, que interfere no processo de captação de energia interferindo no transporte de elétrons do fotossistema II (Dodge, 1971). O imazamox é do grupo químico das imidazolinonas, inibidor da enzima ALS (acetolactato sintase) e é absorvido pela folha e translocado para o xilema e floema causando injúrias e morte das plantas (Tan et al., 2005).

Dentre as plantas aquáticas, a erva do pântano (Enydra sessilis) é classificada como aquática flutuante, perene, decumbente nós radicantes, 15,0 a $40,0 \mathrm{~cm}$ de altura, pouco ramificada. O caule é cilíndrico, fistuloso, estriado, folhoso até o ápice, com ramificações dicotômicas, com folhas a sésseis, obovaladas a oblongas, base obtusa, margem denteada, ápice agudo, membranáceas, glabras, pontuado-glandulosas em ambas as faces, peninérveas (Lima et al., 2006), e sua ocorrência em ambientes aquáticos pode estar relacionada ao aumento da turbidez da água (Carvalho et al., 2005). A Enydra fluctuans também causa interferência no cultivo de arroz (Hasanuzzaman et al., 2009). Para tanto, o objetivo deste estudo foi avaliar a eficácia de controle dos herbicidas imazamox e diquat no controle da erva de pântano (Enydra sessilis), em condição de casa-de-vegetação.

\section{Material e métodos}

Os herbicidas utilizados neste estudo foram o imazamox (670 $\mathrm{g} \mathrm{L}^{-1}$ de ingrediente ativo; e o diquat (200 $\mathrm{g} \mathrm{L}^{-1}$ de diquat). 
O cultivo primário das plantas de Enydra sessilis foi realizado em caixas plásticas com capacidade de 310 litros, preenchidas com substrato formado por areia grossa, material orgânico e solo $\left(1: 1: 1 \mathrm{vv}^{-1}\right)$ e abastecida com sistema de fluxo contínuo de água.

Após o cultivo, foram selecionados três fragmentos apicais de $7 \mathrm{~cm}$ da planta erva do pântano (E. sessilis) que foram transplantados em vasos plásticos com capacidade de $2,5 \mathrm{~L}$, preenchidos com uma mistura de areia grossa, substrato orgânico e solo tipo latossolo $\left(1: 1: 1 ; \mathrm{vV}^{-1}\right)$ e lâmina d'água de $5,0 \mathrm{~cm}$ acima do substrato. Estes foram mantidos em estufa de crescimento por aproximadamente 30 dias, para a aclimatação com temperatura ambiental entre 24,0 e $35,0{ }^{\circ} \mathrm{C}$.

A seguir, foi instalado um experimento para cada herbicida (imazamox e diquat). Os experimentos foram conduzidos em delineamento inteiramente casualizado (DIC), sendo o imazamox testado nas doses de $200,0,400,0,600,0$ e 800 g i.a. ha ${ }^{-1}$ e o diquat a $100,0,200,0,300,0$ e 400,0 g i.a. ha ${ }^{-1}$, com um controle, com dez repetições para cada tratamento.

As aplicações dos herbicidas foram realizadas com um pulverizador costal pressurizado $\left(\mathrm{CO}_{2}\right)$ à pressão constante de $172,36 \mathrm{KPa}$, com consumo de calda de $200 \mathrm{~L} \mathrm{ha}^{-1}$ e equipado com barra de aplicação munida de quatro pontas AD 110.02 da Magnojet ${ }^{\circledR}$, espaçada de $0,5 \mathrm{~m}$ e as condições ambientais no momento das aplicações foram: $25,2{ }^{\circ} \mathrm{C}$ de temperatura, $43,8 \%$ de umidade relativa e vento de 0,3 a $1,2 \mathrm{~km} \mathrm{~h}^{-1}$. Após a pulverização, as plantas foram levadas para casa de vegetação, onde foram mantidas até o final do estudo, com temperatura de 27,0 a $35,0{ }^{\circ} \mathrm{C}$ e umidade relativa do ar de 45 a $60,0 \%$.

A eficácia de controle visual foi realizada por três avaliadores aos 3, 7, 15, 21, 30, 45 e 60 dias após a aplicação (DAA), através de uma escala de notas visuais em porcentagem, em que $0 \%$ corresponde a nenhuma injúria e $100 \%$ à morte das plantas (Sociedade Brasileira da Ciência das Plantas Daninhas, 1995). Ao final do estudo, as plantas foram submetidas a secagem em temperatura ambiente para obtenção da biomassa fresca e transferidas para estufa de circulação forçada de ar, durante três dias a $70{ }^{\circ} \mathrm{C}$, para quantificação da massa seca em balança semianalítica, realizado cálculo de porcentagem simples em relação ao controle.

Os resultados de injúrias foram submetidos à análise de variância (ANOVA) e suas médias, comparadas pelo teste de Tukey a 5\% de probabilidade pelo software Agroestat (Barbosa \& Maldonado Junior, 2015).

\section{Resultados e Discussão}

O herbicida imazamox, na avaliação de 3 dias após aplicação (DAA) não apresentou nenhum sinal de toxicidade visual para a erva do pântano (Enydra sessilis). Em 7 DAA ocorreram pequenas manchas cloróticas nas folhas, correspondendo a um controle em torno de 10,0\% (Tabela 1).

Em 15 e 21 DAA, a eficácia de controle ainda foi insatisfatória, 600,0 g i.a. ha ${ }^{-1}$, diferindo significativamente das demais doses testadas nos dois períodos de avaliação (Tabela 1). O baixo percentual de controle no início do período experimental é devido ao mecanismo de ação do imazamox (inibidor da enzima acetolactato sintase - ALS) (Senseman, 2007).

Em 30 DAA, a melhor eficácia ocorreu com 800,0 g i.a. ha ${ }^{-1}, 83,1 \%$ de controle com necrose dos ponteiros, diferindo das demais doses (Tabela 1). Esta tendência se manteve até o final do período experimental, 6 DAA, com $90,9 \%$ de controle,

Tabela 1. Eficácia (\%) de imazamox no controle de erva do pântano (Enydra sessilis).

\begin{tabular}{|c|c|c|c|c|c|c|c|}
\hline \multirow{2}{*}{ Doses (g i.a. ha ${ }^{-1}$ ) } & \multicolumn{7}{|c|}{ Dias após aplicação (DAA) } \\
\hline & 3 & 7 & 15 & 21 & 30 & 45 & 60 \\
\hline 200,0 & 0,0 & $10,0 \mathrm{a}$ & $11,4 \mathrm{~b}$ & $14,9 \mathrm{~b}$ & $31,5 \mathrm{~b}$ & $62,7 \mathrm{c}$ & $71,6 \mathrm{c}$ \\
\hline 400,0 & 0,0 & $10,0 \mathrm{a}$ & $11,4 \mathrm{~b}$ & $15,5 \mathrm{ab}$ & $32,1 \mathrm{~b}$ & $62,9 \mathrm{c}$ & $72,1 \mathrm{c}$ \\
\hline 600,0 & 0,0 & $10,9 \mathrm{a}$ & $13,5 \mathrm{a}$ & $17,6 \mathrm{a}$ & $30,8 \mathrm{~b}$ & $72,5 \mathrm{~b}$ & $81,0 \mathrm{~b}$ \\
\hline 800,0 & 0,0 & $10,4 \mathrm{a}$ & $11,5 \mathrm{~b}$ & $15,0 \mathrm{~b}$ & $41,1 \mathrm{a}$ & 83,1 a & 90,9 a \\
\hline DMS (5\%) & - & 1,69 & 1,82 & 2,19 & 2,35 & 3,33 & 1,97 \\
\hline $\mathrm{CV}$ & 0,0 & 16,07 & 14,94 & 13,68 & 6,82 & 4,65 & 2,46 \\
\hline $\mathrm{F}$ & - & $121,77 * *$ & $143,94 * *$ & $170,87 * *$ & $722,56 * *$ & $1544,82 * *$ & $5435,88 * *$ \\
\hline
\end{tabular}

Médias seguidas de mesma letra na coluna não diferem estatisticamente entre si pelo teste Tukey $5 \%$; ns = não significativo; $\mathrm{CV}=$ coeficiente de variação. 
sendo esta a melhor concentração em relação à eficácia (Tabela 1). A erva do pântano (E. sessilis) é uma planta prostrada (15 a $40 \mathrm{~cm}$ de altura), com folhas sésseis e pelos epidérmicos em toda a estrutura caulinar e foliar (Lima et al. 2006), o que pode ter diminuído o contato do herbicida imazamox com a planta e sua possível absorção e translocação.

A melhor eficácia obtida no controle de erva do pântano (E. sessilis), em 60 dias após aplicação $\left(800,0 \mathrm{~g}\right.$ i.a. ha $\left.{ }^{-1}\right)$ foi similar à dose de 290,4 $\mathrm{g}$ a.i. ha $^{-1}$ de imazamox com $90,0 \%$ de controle para P. stratiotes em 35 DAA e $83,0 \%$ para Salvinia adnata em 28 DAA e menor que o controle do aguapé (E. crassipes) com 99,5\% em 35 DAA (Campos et al., 2012). Estes autores verificaram que ocorreu diminuição significativa no controle das plantas aquáticas $S$. adnata e $P$. stratiotes, com simulação de chuva 4 horas após aplicação com controle de $67,5 \%$ e $73,7 \%$, respectivamente. A simulação de chuva no estudo de Campos et al. (2012) ou as características morfológicas da erva do pântano (E. sessilis) podem ter diminuído a translocação e a eficácia do imazamox.

Para a $E$. sessilis exposta ao herbicida imazamox na dose de $600,0 \mathrm{~g}$ i.a. ha ${ }^{-1}$, observou-se um controle $30,8 \%$ em 30 DAA, enquanto Garlich et al. (2019) utilizando as doses de 200,0, 400,0 e 600,0 g i.a. ha ${ }^{-1}$ e volume de calda de $100 \mathrm{~L} \mathrm{ha}^{-1}$ para E. crassipes observou uma eficácia de $100,0 \%, 98,70 \%$ e $99,80 \%$, respectivamente, no mesmo período de avaliação.

Chiarotti et al., (2018) em estudo com o herbicida imazamox, na dose de 140,0 $\mathrm{g} \mathrm{ha}^{-1}$ apresentou uma eficácia de 95,0\% em 60 dias após aplicação para a Urochloa subquadripara, diferindo do controle máximo de $81,0 \%$ e $90,9 \%$ da erva de pântano em doses maiores de 600,0 e 800,0 g i.a. ha ${ }^{-1}$ e o mesmo volume de calda aplicado.

Em comparação a um herbicida com outro mecanismo de ação, o imazapyr (inibidor de ALS), na dose de 4,0 L ha ${ }^{-1}$ (1065,2 g i.a ha-1), apresentou $100,0 \%$ de eficácia para $E$. crassipes e P. stratiotes (Chiarotti et al., 2018). A eficácia de controle do imazamox para a erva do pântano também foi menor, mas similar à eficácia de 90,0\% para S. molesta (Cruz et al., 2015a).

Uma alternativa para melhorar a eficácia de herbicidas em controle de plantas aquáticas é a aplicação sequencial que pode resultar em melhores resultados finais. Para Cruz et al., (2017), em condição de mesocosmos, a primeira aplicação de $2880,0,3360,0$ e 3840,0 g i.a. ha ${ }^{-1}$ de glyphosate em três espécies de Salvinias (S. molesta, S. herzogii e $S$. oblongfolia) apresentou variação de controle entre $73,0 \%$ e $85,0 \%$ para $S$. molesta e $S$. herzogii e de 90,0 a 100,0\% para S. oblongfolia em 21 dias após aplicação. Após a segunda aplicação das mesmas doses, o controle atingiu $100,0 \%$ nas três doses para $S$. molesta e $S$. oblongfolia e $95,0 \%$ para $S$. herzogii nas doses de 3360,0 e 3840,0 g i.a. ha $^{-1}$, sendo possível avaliar a evolução do controle. Outras alternativas, segundo Silva et al. (2018), seriam a utilização de adjuvantes para melhorar a deposição da calda de aplicação nas folhas das plantas aquáticas ou tipos de ponta de aplicação, pois para estes autores a utilização de $1,5 \mathrm{~L} \mathrm{ha}^{-1}$ de glyphosate com o Dash $^{\circledR}$ e a ponta TT 110.015 apresentou excelente controle de E. crassipes. Estas poderiam ser alternativas utilizadas para melhorar o controle total da E. sessilis.

O diquat, na primeira avaliação (3 DAA), causou sinais de necrose na Enydra sessilis, em todas as doses testadas, pelo fato de que o diquat é um herbicida de contato. Esses sinais foram evoluindo de acordo com o aumento da concentração. Com 100,0 g i.a. ha ${ }^{-1}$, observou-se $90,4 \%$ de controle e em 400,0 g i.a. ha ${ }^{-1}, 95,9 \%$ de controle. Na avaliação de 7 DAA a eficácia continua sendo considerada excelente para todas as doses testadas e diferindo estatisticamente apenas na dose de 100,0 g i.a. ha ${ }^{-1}$, que apresentou o caule verde, enquanto as folhas já estavam necróticas (Tabela 2). Nas avaliações de 15 a 60 DAA não ocorreu diferença significativa entre as concentrações testadas, todas com um excelente controle (100\%) (Tabela 2).

$\mathrm{Na}$ avaliação de 15 dias após aplicação apresentou $100 \%$ de controle da erva de pântano sem surgimento de rebrotas para todas as doses testadas (Tabela 2). O mesmo herbicida nas doses de 100,0, 200,0,300,0 e 400,0 g i.a. ha ${ }^{-1}$, as mesmas utilizadas no presente estudo, obteve um resultado satisfatório até os 15 dias após a aplicação para a U. subquadripara e o Myriophyllum aquaticum. Depois deste período observou-se o surgimento de rebrota (Cerveira Junior et al. 2019).

Para a E. crassipes, o herbicida diquat na dose de 200 g i.a. ha ${ }^{-1}$ apresentou $63,3 \%$ de controle em 7 dias após aplicação com um volume de calda de $200 \mathrm{~L} \mathrm{ha}^{-1}$ (Almeida et al. 2016), diferindo do controle da erva de pântano, pois nas mesmas 
Tabela 2. Eficácia (\%) de diquat no controle de erva do pântano (Enydra sessilis).

\begin{tabular}{cccccccc}
\hline \multirow{2}{*}{ Doses $\left(\mathbf{g}\right.$ i.a. $\mathbf{~ h a}^{-1}$ ) } & \multicolumn{7}{c}{ Dias após aplicação (DAA) } \\
\cline { 2 - 8 } & $\mathbf{3}$ & $\mathbf{7}$ & $\mathbf{1 5}$ & $\mathbf{2 1}$ & $\mathbf{3 0}$ & $\mathbf{4 5}$ & $\mathbf{6 0}$ \\
\hline 100 & $90,4 \mathrm{~b}$ & $97,1 \mathrm{~b}$ & 100,0 & 100,0 & 100,0 & 100,0 & 100,0 \\
200 & $91,2 \mathrm{~b}$ & $98,4 \mathrm{a}$ & 100,0 & 100,0 & 100,0 & 100,0 & 100,0 \\
300 & $93,9 \mathrm{a}$ & $99,0 \mathrm{a}$ & 100,0 & 100,0 & 100,0 & 100,0 & 100,0 \\
400 & $95,9 \mathrm{a}$ & $99,0 \mathrm{a}$ & 100,0 & 100,0 & 100,0 & 100,0 & 100,0 \\
DMS (5\%) & 2,32 & 0,87 & - & - & - & - & - \\
CV & 2,45 & 0,87 & 0,0 & 0,0 & 0,0 & 0,0 & 0,0 \\
F & $5193,95^{* *}$ & $40904,15^{* *}$ & - & - & - & - & - \\
\hline
\end{tabular}

Médias seguidas de mesma letra na coluna não diferem estatisticamente entre si pelo teste Tukey $5 \%$; ns = não significativo; $\mathrm{CV}=$ coeficiente de variação.

Tabela 3. Massa seca (g) de Enydra sessilis no final do período experimental.

\begin{tabular}{cccc}
\hline Doses $\left(\mathbf{g}\right.$ i.a. $\left.\mathbf{~ h a}^{-1}\right)$ & imazamox & Doses $\left(\mathbf{g}\right.$ i.a. ha $\left.\mathbf{~}^{-\mathbf{1}}\right)$ & diquat \\
\hline 0,0 & $0,012 \mathrm{a}$ & $\mathbf{0 , 0}$ & $0,012 \mathrm{a}$ \\
200,0 & $0,011 \mathrm{a}$ & $\mathbf{1 0 0 , 0}$ & $0,00 \mathrm{~b}$ \\
400,0 & $0,006 \mathrm{a}$ & $\mathbf{2 0 0 , 0}$ & $0,00 \mathrm{~b}$ \\
600,0 & $0,004 \mathrm{a}$ & $\mathbf{3 0 0 , 0}$ & $0,00 \mathrm{~b}$ \\
800,0 & $0,05 \mathrm{a}$ & $\mathbf{4 0 0 , 0}$ & $0,00 \mathrm{~b}$ \\
DMS (5\%) & 0,01 & $\mathbf{D M S ~ ( 5 \% )}$ & 0,01 \\
CV & 107,89 & $\mathbf{C V}$ & 285,48 \\
F & $1,85 \mathrm{~ns}$ & $\mathbf{F}$ & $6,14^{* *}$ \\
\hline
\end{tabular}

Médias seguidas de mesma letra na coluna não diferem estatisticamente entre si pelo teste Tukey 5\%; ns = não significativo; $\mathrm{CV}=$ coeficiente de variação

condições de volume de calda, dose e data de avaliação apresentou um controle de $98,4 \%$ (considerado satisfatório).

Para a massa seca observa-se que o imazamox causou diminuição gradativa com o aumento da dose aplicada, porem não significativa entre a massa final do tratamento controle (Tabela 3). Para o herbicida diquat não foi mensurada a matéria seca devido ao $100 \%$ de controle nos tratamentos testados.

Em relação à massa seca para o herbicida diquat, observa-se que não foi encontrada massa seca mesmo na menor dose testada 100 g i.a. ha ${ }^{-1}$ (Tabela 3), diferindo do estudo de Carbonari et al. (2003), em que o herbicida diquat para o controle de U. subquadripara utilizando a dose de $400 \mathrm{~g}$ i.a. ha $^{-1}$ apresentou $819,7 \mathrm{~g}$ de massa seca ao final do estudo (75 dias após aplicação).

\section{Conclusão}

Conclui-se que o herbicida diquat apresentou um melhor controle para a erva de pântano enquanto o imazamox varia o controle de acordo com a dose utilizada.

\section{Referências}

Almeida, D. P., Agostini, A. R., Yamauchi, A. K., Decaro Junior, S. T., \& Ferreira, M. C. (2016). Application volumes and sizes of droplets for the application of diquat herbicide in the control of Eichhornia crassipes. Planta Daninha, 34(1), 171-179.

Amorim, S. R., Umetsu, C. A., Toledo, D., \& Monteiro Camargo, M. F. A. (2015). Effects of a nonnative species of Poaceae on aquatic macrophyte community composition: A comparison with a native species. Journal of Aquatic Plant Management, 53, 191-196.

Baastrup-Spohr, L., Sand-Jensen, K., Nicolajsen, S. V., \& Bruun, H. H. (2015). From soaking wet to bone dry: predicting plant community composition along a steep hydrological gradient. Journal of Vegetation Science, 26, 619-630.

Barbosa, J. C., \& Maldonado Junior, W. Agroestat: sistema para análises estatísticas e ensaios agronômicos. Jaboticabal: UNESP, 2015. 
Bianchini Junior, I.; Almeida, A. A.; Fushita, A. T., Cunha Santino, M. B., \& Maia, A. T. (2010). Monitoramento das Macrófitas Aquáticas do Reservatório da Usina Hidrelétrica Luís Eduardo Magalhães (Estado de Tocantins, Brasil). Augmdomus, (2), 38-48.

Campos, C. F., Souza, G. S. F., Martins, D., Pereira, M. R. R., \& Bagatta, M. V. B. (2012). Influência da chuva após aplicação de imazamox sobre o controle de plantas daninhas aquáticas. Bioscience Journal, 28(3), 413-419.

Carbonari, C. A., Martins, D., \& Terra, M. A. (2003). Control of Brachiaria subquadripara and Brachiaria mutica by different herbicides applied post-emergence. Planta Daninha, 21(n. spe), 79-84.

Carvalho, F. T., Velini, E. D., Cavenaghi, A. L., Negrisoli, E., \& Castro, R. M. (2005). Influência da turbidez da água do rio tietê na ocorrência de plantas aquática. Planta Daninha, 23(2), 359-362.

Cerveira Junior, W. R., Silva, A. F., Cruz, C., Pitelli, R. A., \& Martins, D. (2019). Herbicides for rooted aquatic macrophytes. Planta Daninha, $37, \mathrm{e} 019175938$.

Chiarotti, L. F., Pereira, P. C., Scannavino, R. H. D. C. P., Castro, K. S., Junior, W. R. C., \& Da Cruz, C. (2018). Eficácia de herbicidas isolados ou em mistura no controle da planta exótica invasora Urochloa subquadripara. Revista Brasileira de Herbicidas, 17(2), 596-1.

Conselho Nacional do Meio Ambiente-CONAMA. (2015, 17 de julho). Resolução ${ }^{\circ}$ 467, de 16 de julho de 2015. Dispõe sobre critérios para a autorização de uso de produtos ou de agentes de processos físicos, químicos ou biológicos para o controle de organismos ou contaminantes em corpos hídricos superficiais e dá outras providências. Diário Oficial [da] República Federativa do Brasil, Brasília. Seção 1, p. 70-71.

Cruz, C., Cervoni, J. H. C., Silva, A. F., Garlich, N., \& Pitelli, R. A. (2017). Aplicação sequencial de glyphosate em plantas aquáticas de difícil controle em condição de mesocosmos. Ciência e Cultura, 13(2), 59-64.

Cruz, C., Silva, A. F., Luna, L. V., Yamauchi, A. K. F., Garlich, N., \& Pitelli, R. A. (2015b).
Glyphosate Effectiveness in the Control of Macrophytes Under a Greenhouse Condition. Planta Daninha, 33, 241-247.

Cruz, C., Silva, A. F., Shiogiri, N. S., Garlich, N., \& Pitelli, R. A. (2015a). Imazapyr herbicide efficacy on floating macrophyte control and ecotoxicology for non-target organisms. Planta Daninha, 33(1), 103-108.

Dodge, A. D. (1971). The mode of action of the bipyridylium herbicides, paraquat and diquat. Endeavor, 30, 130-135.

Garlich, N., Guarnieri, C. C. O., Freitas, R. L. G., Cervoni, J. H. C., Cruz, C., \& Ferreira, M. C. (2019). Efficacy of imazamox with centrifugal energy spray nozzle on Eichhornia crassipes and economic analysis of control viability. Planta Daninha, 37, 1-8.

Gettys, L. A., Haller, W. T., \& Petty, D. G. (2014). Biology and control of aquatic plant: $A$ best management practices handbook. Marietta, GA: Aquatic Ecosystem Restoration Foundation.

Hasanuzzaman, M., Ali, M. H., Alam, M. M., Akther, M., \& Fakhrul Alam, K. F. (2009). Evaluation of preemergence herbicide and hand weeding on the weed control efficiency and performance of transplanted aus rice. American-Eurasian Journal of Agronomy, 2(3), 138-143.

Hofstra, D. E., Champion, P. D., \& Dugdale, T. M. (2006). Herbicide trials for the control of parrotsfeather. Journal of Aquatic Plant Management, 44, 13-18.

Keddy, P. A. (1992). Assembly and response rules: two goals for predictive community ecology. Journal of Vegetation Science, 3, 157-164.

Lima, L. F. P., Schneider, A. A., \& Matzenbacher, N. I. (2006). Nota sobre a ocorrência de Enydra sessilis (SW.) DC. (Asteraceae-Heliantheae) para o estado do Rio Grande do Sul, Brasil. Pesquisas, série Botânica, 57, 153-156.

Liu, C., Xie, G., \& Huang, H. (2006). Shrinking and drying up of Baiyangdian Lake wetland: A natural or human cause? Chinese Geographical Science, 16, 314-319. 
Mudge, C. R., \& Netherland, M. D. (2014). Silva, A. F., Cerveira Junior, W. R., Ferreira, M. C., Response of giant bulrush, water hyacinth, and \& Cruz, C. (2018). Floating macrophyte control water lettuce to foliar herbicide applications. with glyphosate according to adjuvants and spray Journal of Aquatic Plant Management, 52, nozzles. Planta Daninha, 36, 1-8. 75-80.

Richardson, R. J., Haug, E. J., \& Netherland, M. Sociedade Brasileira da Ciência das Plantas Daninhas - SBCPD. (1995). Procedimentos para D. (2016). Response of seven aquatic plants to a new arylpicolinate herbicide. Journal of Aquatic Plant Management, 54, 26-31.

Senseman, S. A. (2007). Herbicide handbook (9th ed.). Lawrence, EUA: Weed Science Society of America Lawrence. instalação, avaliação e análise de experimentos com herbicidas (Vol. 42). Londrina: SBCPD.

Tan, S., Evans, R. R., Dahmer, M. L., Singh, B. K., \& Shaner, D. L. (2005). Imidazolinone-tolerant crops: history, current status and future. Pest Management Science, 61(3), 246-257. 